\title{
Velocity-Controlled Piezoelectric Switching Energy Harvesting Device
}

\author{
Yuan-Ping Liu ${ }^{1,2}$, Dejan Vasic ${ }^{1}$, François Costa ${ }^{1,3}$, Wen-Jong Wu ${ }^{2, *}$, C. K. Lee $2,4,5$ \\ ${ }^{1}$ Système et Application des Technologies de l’Information et de l'Energie, UNIVERSud, Ecole Normale Supérieure de \\ Cachan, France \\ ${ }^{2}$ Department of Engineering Science and Ocean Engineering, National Taiwan University, Taipei, Taiwan \\ ${ }^{3}$ IUFM de Créteil, Université Paris 12, Place du 8 mai 1945, 93000 St Denis, France \\ ${ }^{4}$ Institute of Applied Mechanics, National Taiwan University, Taiwan \\ ${ }^{5}$ Industrial Technology Research Institute of Taiwan, Taiwan \\ Address: No.1, Sec. 4, Roosevelt Rd., Da-an District, Taipei City 106, Taiwan \\ Phone/Fax number: +886 23366 5764, e-mail: ypliu@mems.iam.ntu.edu.tw
}

\begin{abstract}
The main purpose of this paper is to design the interface circuit of the piezoelectric energy harvesting device which can be suitable for a resistive load o a capacitive load. The vibrating velocity is detected to control the active rectifier to make the power factor correction of the piezoelectric layers. However, there are switching losses in the active rectifier in practice. The dead time and an inductor are thus taken into consideration to minimum the switching loss. The proposed circuit can increase 4 times than typical interface circuit of the piezoelectric layer, and can increase the charging speed of the capacitive storage buffer as well.
\end{abstract}

\section{Key words}

Piezoelectric layers, power factor correction, power harvesting, capacitive storage

\section{Introduction}

The piezoelectric energy harvesting device can be identified as two main parts. The first part is the piezoelectric material itself. The improvement of electromechanical coupling and piezoelectric configurations are the main issues in this research line. The other part is the interface circuit design. Different interface circuit may influence efficiency of the energy harvesting device very much. In this paper, we focused on the interface circuit design to propose an optimal and realizable harvesting circuit.

As to the harvesting interface circuit, the first issue is how to store the energy. There are many storage methods. Rechargeable batteries are an option for this energy storage purpose. Different rechargeable batteries have different charging characteristics and also perform differently when connected to an energy harvesting power source. $\mathrm{Ni}-\mathrm{Cd} / \mathrm{Ni}-\mathrm{Mn}$ batteries can be simply treated as a resistive load, which were adopted in most studies when used with energy harvesting power sources. However, rechargeable batteries have notorious memory effects and have limited charge/discharge endurance. Super (ultra) capacitors are another promising energy storage buffer. As their technology improves and their cost drops, super capacitors are now replacing small button batteries for memory backup applications. Unfortunately, the properties of super capacitors are much different from those of rechargeable batteries. Super capacitors are characterized as reactive loads as opposed to resistive loads and have many shortcomings such as low sustained voltage and high leakage. The optimization of the power output from an energy harvesting source is much different for reactive loads and resistive loads. The charging and interfacing circuit for an ultra-capacitor must be designed differently from one for a rechargeable battery. On the other hand, the piezoelectric layer itself also owns highly capacitive characteristics. Under short circuit state and the open circuit state, piezoelectric layer only generates the reactive power but not real power. The reactive power cannot be stored due to the power factor is equal to zero. The best way is to make the interface circuit and the piezoelectric layer become resistive but not reactive. In other words, the interface circuit must have the function of the power factor correction. Based on these problems and difficulties above, the objective of this paper is to make an innovative interface circuit to solve the problem of highly capacitive characteristics of both piezoelectric layers and the super-capacitor.

There are many researches discussed about the interface circuit of the piezoelectric harvesting device. The most popular techniques are called the synchronized switching harvesting devices. [1, 2] Synchronized switching techniques indeed improve the efficiency of the piezoelectric harvesting device a lot. However, the storage buffers in most synchronized switching techniques are considered as a resistive load but not a capacitor. $\mathrm{Wu}$ [3] thus proposed synchronized switching techniques with capacitive storage buffer to solve this problem. However, the interface circuit still have the 
possibility to improve. Liu [4] developed the velocitycontrolled switching circuit for piezoelectric damping. This technique is based on the work cycle observation of the piezoelectric layer and the concept of the power factor correction. Actually, the piezoelectric damping and energy harvesting device are similar. The most difference is that the piezoelectric damping takes the energy from the piezoelectric layer to dissipate, but the piezoelectric energy harvesting device takes the energy from the piezoelectric layer to make storage. More specifically, both of these two devices take energy from the piezoelectric layer. Therefore, the interface circuit designs of damping and harvesting devices are similar. It should be noted that the concept of Liu's velocitycontrolled switching circuit has been proved as an optimal interface circuit for the piezoelectric damping. [4] However, both the switching loss and pure capacitive load were not considered in Liu's velocity-controlled circuit. However, both these two issues are important in the energy harvesting device. Therefore, this paper focused on these two issues and extended Liu's work to the piezoelectric harvesting device with both resistive load and the capacitive storage buffer.

\section{The Circuit Topologies}

The piezoelectric harvesting system is shown in the Fig. 1. A piezoelectric layer is adhered to a structure. An interface circuit is connected to the piezoelectric layers to take and convert the energy to resistive load or the capacitive storage buffer. Normally, the maximum energy flows out of the piezoelectric layer at the fundamental resonant frequency of the structure. The alternating current flows out the piezoelectric layer. On the other hand, the storage buffer is to store the DC voltage. Therefore, the interface circuit is an AC/DC converter.

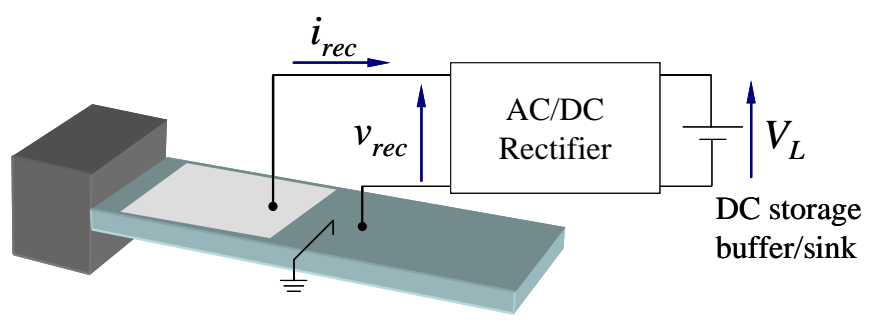

Fig 1. The basic configuration of the piezoelectric based harvesting device

The basic interface circuit is a full-wave rectifier. The equivalent circuit of the piezoelectric layers with the full-wave rectifier is shown in Fig 2. The current source in Fig. 2 represents the mechanical velocity. The voltage waveform and the current waveform flowing out of the piezoelectric layers are shown in Fig. 3. It should be noted that the current flowing into the rectifier is not continuous. This fact leads the energy cannot be efficiently taken from the piezoelectric layers. The discontinuous current is caused from the charging of the piezoelectric capacitor. More specifically, the rectifier only conducted when the rectifier voltage is equal to the load voltage. However, the piezoelectric capacitor is required to charge and discharge in each period. In the period of the piezoelectric capacitor charging, the voltage of the piezoelectric capacitor is smaller than the load voltage, and the current do not flow into rectifier. Once the piezoelectric capacitor is charged at the load voltage, the rectifier starts to conduct and the current passes through the rectifier to the load. This charging time leads that the rectifier voltage and the rectifier current are not in phase. The phase difference causes the power not only flows from the piezoelectric layer to the load but also flows back from the load to the piezoelectric layer. More specifically, the power factor is not yet correction.

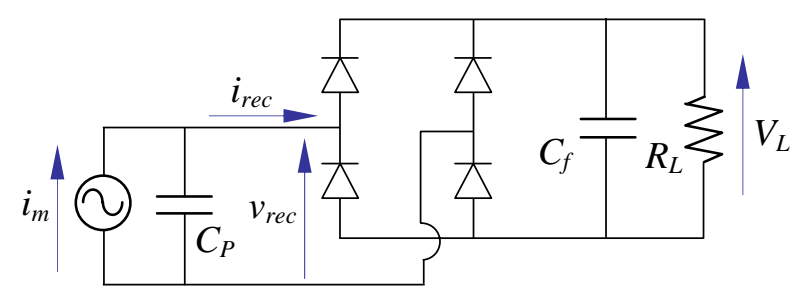

Fig 2. The full-wave rectifier

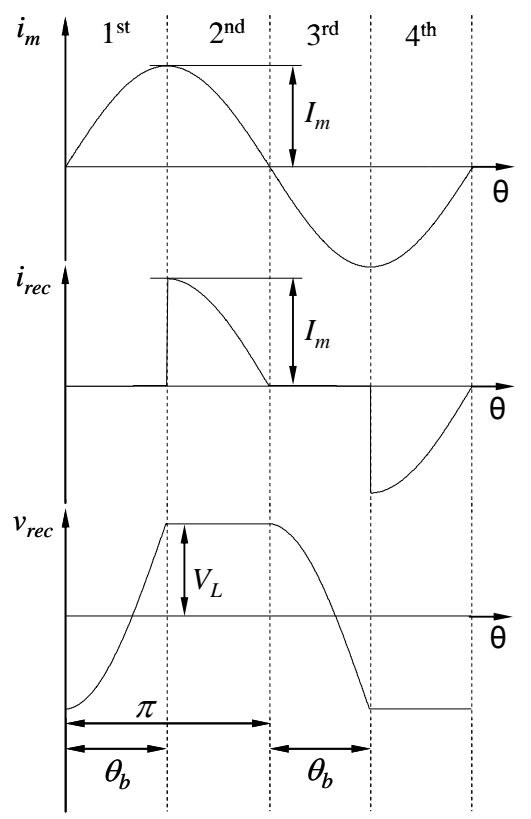

Fig 3. The piezoelectric layer fed full-wave rectifier

To make the power factor correction, the fundamental component of the mechanical current and the piezoelectric voltage have to be in phase. To achieve this goal, four switches are replaced four diodes to change the phase of the piezoelectric output voltage (see Fig. 4). The piezoelectric sensor with current amplifier is used to detect the mechanical current (velocity) of the piezoelectric layer. When the mechanical current is positive, the switches $\mathrm{L} 1$ and R2 are turned $\mathrm{ON}$ and the switches L2 and R1 are turned OFF (Fig. 5 (a)). The piezoelectric voltage is thus positive in this stage. When the mechanical current is negative, the switches L1 and $\mathrm{R} 2$ are turned $\mathrm{ON}$ and the switch L2 and R1 are turned OFF (Fig. 5(b)). The piezoelectric voltage is thus negative in this period. Accordingly, the mechanical current and the piezoelectric voltage become in phase. The ideal waveforms are shown in Fig. 6. However, this circuit topology is not suitable for the harvesting device 
in practice. The underlying reason is that the switches cause the piezoelectric capacitor and the parasitic capacitor of the switch become short circuit in the short switching period. The switching loss is thus generated significantly.

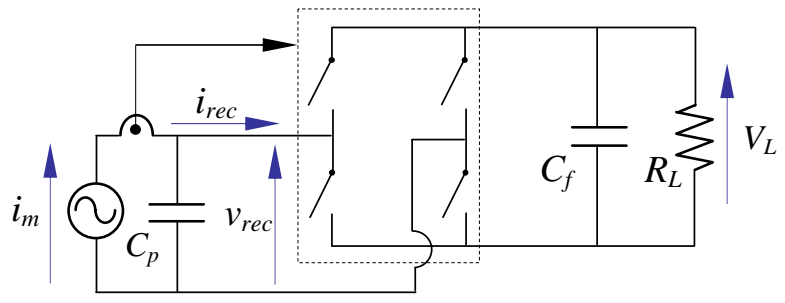

Fig 4. Velocity-controlled piezoelectric switching energy harvesting device

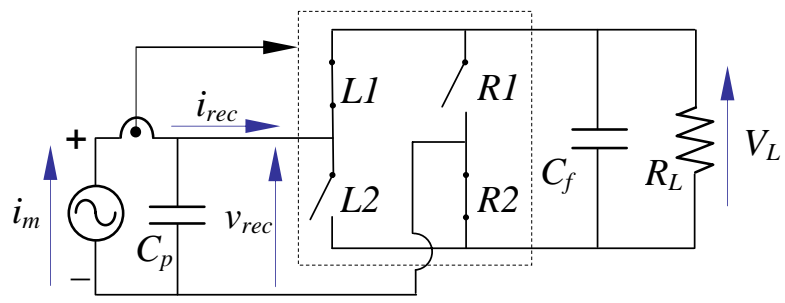

(a)

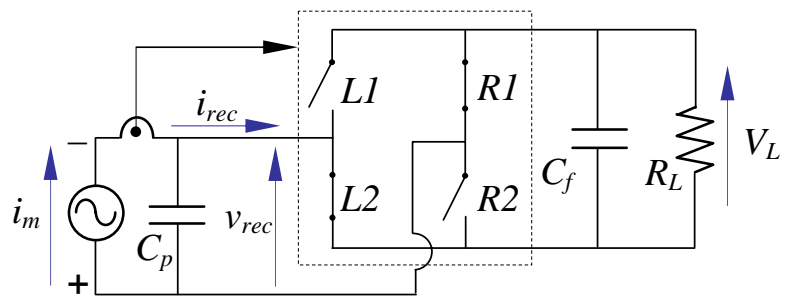

(b)

Fig 5. The operating state of (a) the positive current and (b) the negative current

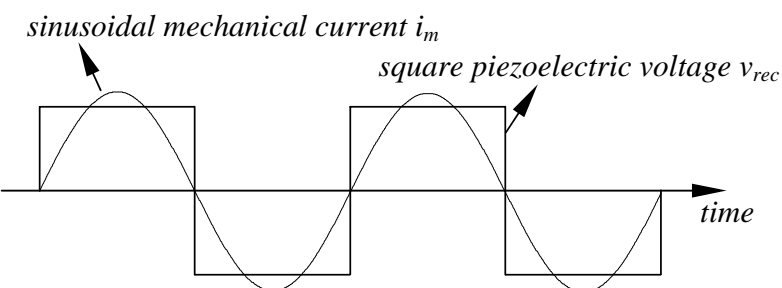

Fig 6. The waveform of the ideal VSPH system

To minimize the practical switching loss, the piezoelectric capacitor must be full charged before the switching action. To make the piezoelectric capacitor fully charge faster, an inductor is added between the switching stage and the piezoelectric capacitor. In addition, the diode is added to ensure the current is flowing from piezoelectric layer to the load but not from the load to the piezoelectric layer. Specifically, this diode only conducts when the piezoelectric voltage is equal to the load voltage. The improved circuit of VSPH is shown in Fig. 7. The operating law is shown in Fig. 8. Same as the basic thinking of the VSPH, the switches L1 and R2 are turned on and the switches L2 and R1 are turned off when the mechanical current is positive (see Fig. 8(a)).
The switches L1 and R2 are turned off and the switches L2 and R1 are turned on when the mechanical current is negative (see Fig. 8(c)). However, between these two states, the four switches are turn off, which is called "dead time". During the dead time the piezoelectric capacitor charged with the series inductor, the piezoelectric capacitor is thus charged very fast. Fig. 9 shows the waveform during the dead time. In can be seen an impulse current is charging the piezoelectric capacitor. This is the key point of the VSPH to implement the square voltage with sinusoidal current. On the other hand, the optimal value of the series inductor is relative to the time constant.

$$
t_{d}=\frac{\pi}{2} \sqrt{L_{s} C_{p}}
$$

where $t_{d}$ is the dead time. We can set the short dead time to obtain the small series inductance and to make the piezoelectric capacitor charge faster. However, the larger impulse current may induce. In practice, the mechanical current is small as several micro-amperes, so it is not difficult to set the short dead time and add very small inductor. In fact, the waveform of the improved VSPH also followed the waveform in Fig. 6. The difference is only the charging and discharging of the piezoelectric capacitor in the dead time.

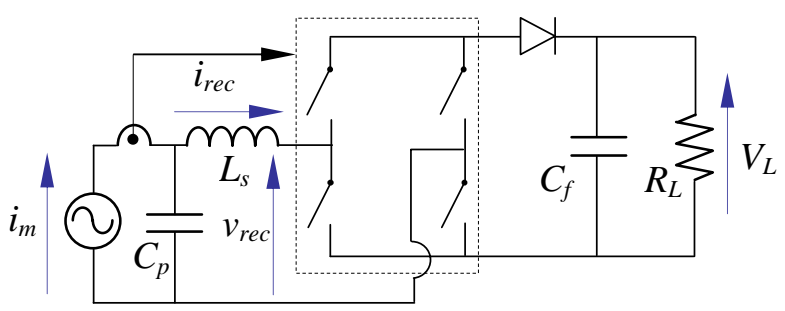

Fig 7. Velocity-controlled switching piezoelectric Energy harvesting Device

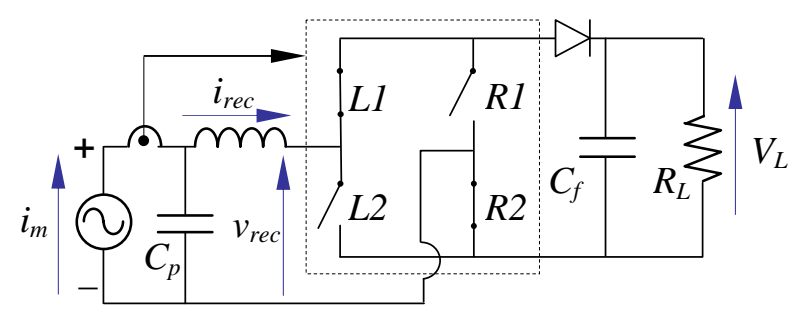

(a)

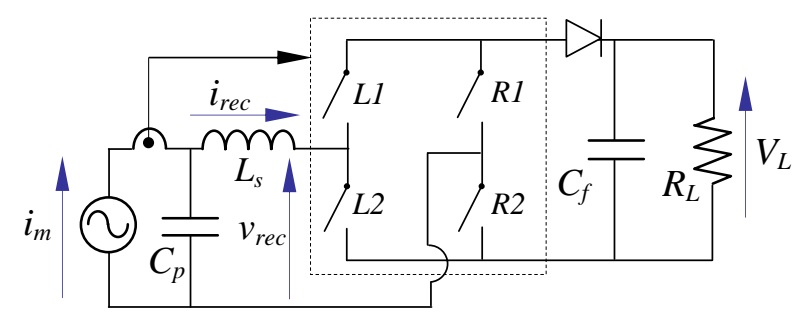

(b) 


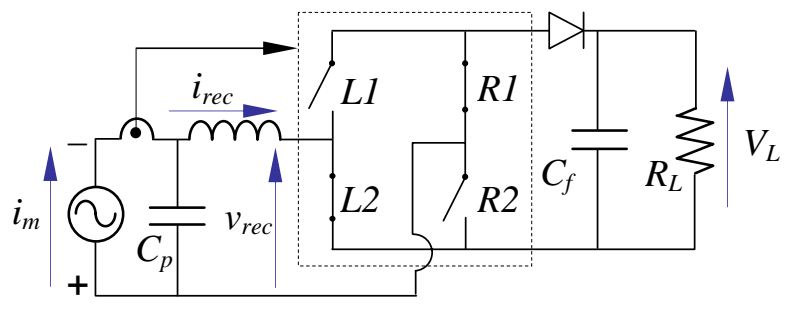

(c)

Fig 8. The operation of the VSPH: the states of (a) positive mechanical current (b) dead time (c) negative mechanical current

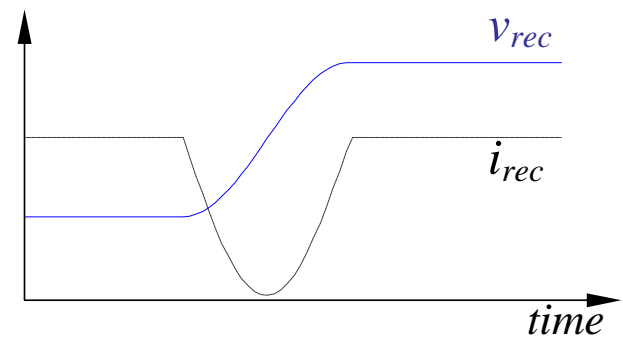

Fig 9. The rectifier voltage $v_{\text {rec }}$ (solid line) and the rectifier current (dot line) of the modified VSPH

\section{Comparisons}

There are two points of interests for the harvesting device, which are the charging speed of the storage buffer and the total energy we obtained. The proposed VSPH system is compared with the typical rectifier in this paper. In fact, the typical harvesting circuit, i.e. full-wave rectifier, has been fully developed [3, 4].The load voltage is:

$$
V_{L}^{t y p}=\frac{2 R_{L}}{\pi+2 \omega C_{p} R_{L}} I_{m}
$$

And the corresponding optimal load is

$$
R_{L}^{*}=\frac{\pi}{2 \omega C_{p}}
$$

As to VSPH, the analysis is simpler since the piezoelectric voltage and the mechanical current is in phase. According to the energy conservation, the power from the source is equal to the load power, such as:

$$
\frac{1}{\pi} \int_{0}^{\pi} V_{L} I_{m} \sin \theta d \theta=\frac{V_{L}^{2}}{R_{L}}
$$

The load voltage can be solved based on Eq. (4):

$$
V_{L}=\frac{2 R_{L}}{\pi} I_{m}
$$

In should be noted that the load voltage of the VSPH is independent of the piezoelectric capacitor. There are two loads of interests. The first case is the optimal resistive load, i.e. Eq. (4). Substituting Eq. (4) into Eq. (3), the load voltage of the typical rectifier leads that:

$$
V_{L}^{t y p}=\frac{R_{L}}{\pi} I_{m}
$$

It should be noted that the voltage in Eq. (6) is two times smaller than the voltage of VSPH in Eq. (5). In other words, the power from the VSPH is 4-times larger than the typical rectifier in the steady state. The simulation result is shown in Fig. 10.

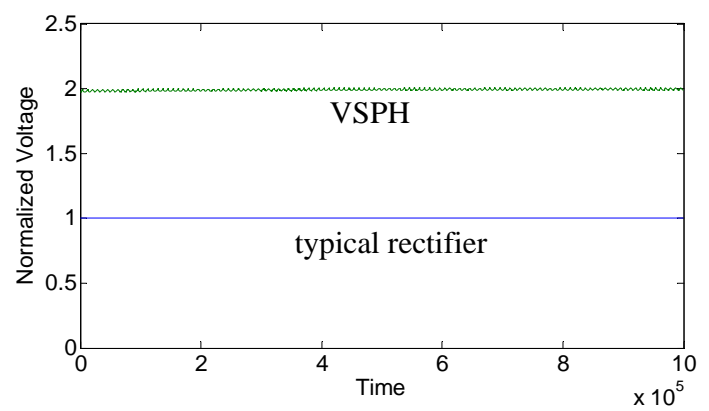

Fig 10. The normalized load voltage of the typical rectifier and the VSPH (steady state with resistive load)

Another case of interest is the capacitive storage buffer, i.e. the resistive load is infinite. In this case, different from the resistive load, the load voltage may never achieve steady state especially when the super capacitor is used as the storage buffer. The transient state is thus more important than the steady state. In such a case, the faster charging speed is better. However, the transient state is difficult to solve analytically. The simulation is applied to both the typical rectifier and the VSPH. The result of the transient state is shown in Fig. 11. Obviously, the VSPH is still better than typical rectifier.

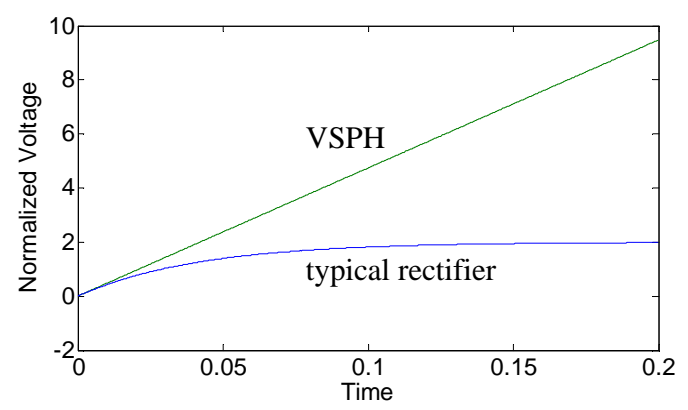

Fig 11. The normalized load voltage of the typical rectifier and the VSPH (transient state with capacitive load)

\section{Conclusion}

This paper proposed an innovative interface circuit for the piezoelectric energy harvesting device based on the power factor correction. The proposed circuit was called velocity-controlled piezoelectric switching energy harvesting device (VSPH). An active rectifier is controlled by the velocity signal to put square piezoelectric voltage and the sinusoidal mechanical velocity in phase to obtain the best power factor. In 
addition, the dead time and an inductor were also introduced to decrease the switching loss; a diode was added to ensure the energy is only flowing from the piezoelectric layer to the load. The results show that the proposed VSPH circuit can increase 4 times larger power with resistive load in the steady state and has better charging speed in the transient state with the capacitive storage buffer. Therefore, the VSPH can make the application of the piezoelectric harvesting device more close to the practical case.

\section{Acknowledgment}

The authors are grateful to ELECERAM TECHNOLOGY Co., LTD. for continuously providing us with all different types piezoelectric layers used in the research work.

\section{References}

[1] D. Guyomar, A. Badel A, E. Lefeuvre and C. Richard, "Toward Energy Harvesting Using Active Materials and Conversion Improvement by Nonlinear Processing”, IEEE Trans. Ultrason. Ferroelectr. Freq. Control, Vol. 52, pp. 584-595, 2005.

[2] E. Lefeuvre, A. Badel, C. Richard and D. Guyomar, ” Piezoelectric Energy Harvestin Device Optimization by Synchronous Electric Charge Extraction”, J. Intell. Mater. Syst. Struct., Vol. 16 pp. 865-876, 2005.

[3] W. J. Wu, A. Wickenheiser, T. Reissman, and E. Garcia, "Modeling and Experimental Verification of Synchronized Discharging Techniques for Boosting Power Harvesting from Piezoelectric Transducers", Smart Materials and Structures (accepted).

[4] Y. P. Liu, D. Vasic, F. Costa, W. J. Wu, C. K. Lee, "Velocity-Controlled Switching Piezoelectric Damping Based on Maximum Power Factor Tracking and Work Cycle Observation", Proceedings of 19th International Conference on Adaptive Structures and Technologies (ICAST 2008), paper no. 40, Ascona, Switzerland, October 6-9, 2008. 